\title{
CR-HYPERSURFACES OF THE SIX-DIMENSIONAL SPHERE
}

\author{
M.A. BASHIR \\ Mathematics Department, College of Science \\ King Saud University, P.O. Box 2455 \\ Riyadh 11451, Saudi Arabia
}

(Received July 23, 1992)

ABSTRACT. We proved that there does not exist a proper $C R$-hypersurface of $S^{6}$ with parallel second fundamental form. As a result of this we showed that $S^{6}$ does not admit a proper $C R$-totally umbilical hypersurface. We also proved that an Einstein proper $C R$-hypersurface of $S^{6}$ is an extrinsic sphere.

KEY WORDS AND PHRASES. Nearly Kaehler manifold, $C R$-submanifold, six-dimensional sphere, Einstein hypersurface totally umbilical.

1991 AMS SUBJECT CLASSIFICATION CODES. 53C40, 53C55.

\section{INTRODUCTION.}

It is known that of all the Euclidean spheres $S^{2}$ and $S^{6}$ admit the almost complex structure of which $S^{2}$ is complex and $S^{6}$ is not. It is also known that $S^{6}$ is an almost hermitian manifold which is nearly Kaehler but not Kaehler [4], that is, the almost complex structure is not parallel with respect to the Riemannian connection on $S^{6}$. Among all submanifolds of an almost Hermitian manifold, there are three typical classes; one is the class of holomorphic submanifold, one is the class of totally real submanifolds and the third is the class of $C R$-submanifolds. This last class was introduced by Bejancu [1]. Let $(\bar{M}, J, g)$ be an almost Hermitian manifold with almost Hermitian structure $(J, g)$ and $M$ be a Riemannian submanifold of $\bar{M}$. The $M$ is called a $C R$-submanifold of $\bar{M}$ if there exists a $C R$-holomorphic distribution $D$, i.e., $J D=D$ on $M$ such that its orthogonal complement $D^{\perp}$ is totally real, i.e., $J D^{\perp} c \nu$ where $\nu$ is the normal bundle over $M$ in $\bar{M}$. A $C R$-submanifold is called proper if neither $D=0$, nor $D^{\perp}=0$. The three classes of submanifolds of $S^{6}$, including $C R$-submanifolds, have been studied by several authors [2], [3], [5]. In this paper, we consider $C R$-hypersurfaces of $S^{6}$. We obtain the following results:

THEOREM 1. There does not exist a proper $C R$-hypersurface of $S^{6}$ with parallel second fundamental form.

THEOREM 2. $S^{6}$ does not admit a proper $C R$-totally umbilical hypersurface.

THEOREM 3. Let $M$ be an Einstein proper $C R$-hypersturface of $S^{6}$, then $M$ is an extrinsic sphere.

PRELIMINARIES. Let $(\bar{M}, g)$ be a Riemannian manifold and $M$ be a Riemannian submanifold of $\bar{M}$. Let $\nabla$ (resp. $\bar{\nabla}$ ) be the Riemannian connection on $M$ (resp. $\bar{M}$ ) and $R$ (resp. $\bar{R}$ ) be the curvature tensor of $M$ (resp. $\bar{M}$ ). Denote by $h$ the second fundamental form of $M$ in $\bar{M}$. Then the Gauss formula and the Weingarten formula are given respectively by

$$
\bar{\nabla}_{X} Y=\nabla_{X} Y+h(X, Y)
$$




$$
\bar{\nabla}_{X} N=-A_{N} X+\frac{1}{\nabla} X^{N} \quad \underset{N \in \nu}{X, Y \in G(M)}
$$

where $-A_{N} X$ (resp. $\frac{1}{\nabla}{ }_{X} N$ ) denotes the tantial part (resp. normal part) of $\bar{\nabla}_{X^{N}}$. The tangential component $A_{N} X$ is related to the second fundamental form by

$$
g(h(X, Y), N)=g\left(A_{N} X, Y\right), \quad X, Y \in \mathscr{S}(M) .
$$

The Gauss equation is given by

$$
g(R(X, Y) Z, W)=g(\bar{R}(X, Y) Z, W)+g(h(X, W), h(Y, Z))-g(h(X, Z), h(Y, W))
$$

The Codazzi equation is

where

$$
\begin{gathered}
g(\bar{R}(X, Y) Z, N)=g\left(\left(\bar{\nabla}_{X} h\right)(Y, Z)-\left(\bar{\nabla}_{Y} h\right)(X, Z), N\right) \\
\left(\bar{\nabla}_{X} h\right)(Y, Z)=\bar{\nabla}_{X} h(Y, Z)-h\left(\nabla_{X} Y, Z\right)-h\left(Y, \nabla_{X} Z\right)
\end{gathered}
$$

If $\left(e_{i}\right) i=1,2, \cdots, \eta$ is a frame field for $M$, then the Ricci curvature $S$ of $M$ is given by

$$
S(X, Y)=\sum_{i=1}^{n} R\left(e_{i}, X, Y, e_{i}\right) .
$$

The submanifold $M$ is called an Einstein manifold if $S(X, Y)=c g(X, Y)$ for some constant $c$ and any $X, Y \in \mathscr{S}(M) . \quad M$ is said to be totally umbilical if $h(X, Y)=g(X, Y) H$ where $H$ is the mean curvature vector defined by $H=\frac{1}{n}$ trace $h$.

$M$ is called an extrinsic sphere if $\frac{i}{\nabla} X_{X} H=0$ for any $X \in \mathscr{S}(M)$. The $C R$-submanifold $M$ is called a $C R$-product submanifold if it is locally the Riemannian product of a holomorphic submanifold and a totally real submanifold. Sekigawa [6] proved that in $S^{6}$ there does not exist any $C R$-product submanifolds.

2. PROOF OF THE MAIN RESULTS.

PROOF OF THEOREM 1 . Since the second fundamental form is parallel we have $\left(\nabla_{W} A\right)(V)=0$ or $\nabla_{W} A V=A \nabla_{W} V$ for any $V, W \in \Phi(M)$. If $V$ is an eigenvector of $A$ with corresponding eigenvalue $\beta$, i.e., $A V=\beta V$, then from the equation $\nabla_{W}(A V)=A \nabla_{W} V$ we get $\beta \nabla_{W} V=A \nabla_{W} V$. This means that $\nabla_{W} V$ is an eigenvector corresponding to eigenvalue $\beta$ whenever $V$ is. If $T$ is the eigenspace of $\beta$ then $\nabla_{W} T c T$.

Since $M$ is a proper $C R$-hypersurface of $S^{6}$, we can take $\left\{E_{1}, J E_{1}, E_{2}, J E_{2}, \xi\right\}$ as an orthonormal frame field for $T M$ where $E_{1}, E_{2} \in D$ and $\xi \in D^{\perp}$. Also since the normal bundle is 1dimensional we assume that the frame $\left\{E_{1}, J E_{1}, E_{2}, J E_{2}, \xi\right\}$ diagonalizes $A$. So let $A E_{1}=\alpha_{1} E_{1}$, $A J E_{1}=\bar{\alpha}_{1} J E_{1}, A E_{2}=\alpha_{2} E_{2}, A J E_{2}=\bar{\alpha}_{2} J E_{2}$ and $A \xi=\beta \xi$. We consider the two cases for the eigenvalues $\alpha_{i}, \bar{\alpha}_{i}, \beta \quad i=1,2$.

CASE 1: $\alpha_{i} \neq \beta$ and $\bar{\alpha}_{i} \neq \beta$ for all $i=1,2$.

In this case we have $g\left(\nabla_{W} \xi, E_{i}\right)=g\left(\nabla_{W} \xi, J E_{i}\right)=0$ for all $W \in \mathscr{S}(M)$. This gives $\nabla_{W} \xi \in D^{\perp}$, i.e., the distribution $D^{\perp}$ is parallel. Since $\nabla_{W} \xi \in D$ we get $\nabla_{W} \xi=0$. This last equation with $g(\xi, X)=0$ for $X \in D$ gives $\nabla_{W} X \in D$, i.e., the distribution $D$ is also parallel. This implies that $M$ is a $C R$-product, a contradiction, since $S^{6}$ does not admit any $C R$-product submanifold [6].

CASE 2: $\alpha_{i_{o}}=\beta$ or $\bar{\alpha}_{i_{o}}=\beta$ for some $i_{o}$.

Without loss of generality let us assume that $\alpha_{i_{o}}=\beta$ for some $i_{o}$. Then the space $T$ spanned by $\left\{E_{i_{o}}, \xi\right\}$ is the eigenspace of eigenvalue $\beta=\alpha_{i_{o}}$. We then have $\nabla_{W} T$. In particular 
$\nabla_{E_{i}} \xi=a E_{i}+b \xi$ for some functions $a$ and $b$. Since $g\left(\nabla_{E_{I}} \xi, \xi\right)=0$, we get $\nabla_{E_{i}} \xi=a E_{i_{o}}$. Also using the equation $\bar{\nabla}_{E_{i_{o}}^{J E_{o}}}=J \bar{\nabla}_{E_{i_{o}}} E_{i_{o}}$ with the help of equations (1.1) and (1.2) and the fact that $h \in J D^{\perp}$ we get $g\left(\nabla_{E_{i_{o}}} E_{i_{o}}, \xi\right)=0$. From which we get $g\left(\nabla_{E_{i_{o}}} \xi, E_{i_{o}}\right)=0$, i.e., $\nabla_{E_{i_{o}}} \xi=0$. Now using this last equation and the fact that $\nabla_{\xi} \xi=0$, we get

$$
R\left(E_{i_{o}}, \xi\right) \xi=\nabla_{E_{i_{o}}} \nabla_{\xi} \xi-\nabla_{\xi} \nabla_{E_{i_{o}}}{ }^{\xi}-\nabla_{\left[E_{i_{o}}, \xi\right]} \xi=\underset{\xi}{\nabla} E_{i_{o}}^{\nabla}
$$

But $\nabla_{\xi} E_{i_{o}}=c E_{i_{o}}+d \xi=0$ since $g\left(\nabla_{\xi} E_{i_{o}}, E_{i_{o}}\right)=0 . \quad g\left(\nabla_{\xi} E_{i_{o}}, \xi\right)=-g\left(\nabla_{\xi} \xi, E_{i_{o}}\right)=0$. So $R\left(E_{i_{o}}, \xi\right) \xi=0$. However from Gauss equation we obtain $g\left(R\left(E_{i_{o}}, \xi\right) \xi, E_{i_{o}}\right)=c+\beta^{2}>0$ which is a contradiction. This finishes the proof of Theorem 1.

PROOF OF THEOREM 2. Since $M$ is totally umbilical we have $h(X, Y)=g(X, Y) H$ for any $X, Y \in \mathscr{G}(M)$. Using this in Codazzi equation (1.4) we get $g(R(X, Y) Z, N)=g\left(g(Y, Z)^{\frac{1}{\nabla}} X^{H}\right.$ $\left.-g(X, Z) \frac{1}{\nabla} Y^{H, N}\right)$. Since the ambient space $S^{6}$ is of constant curvature we have $g\left(g(Y, Z) \stackrel{1}{\nabla}_{X} H-g(X, Z) \stackrel{1}{\nabla}_{Y} H, N\right)=0, X, Y, Z \in \mathscr{S}(M)$. Now for any $X \in \mathscr{S}(M)$, choose $Y$ such that $g(Y, X)=0$ and let $Z=Y$. Then the above equation gives ${ }_{\nabla}{ }_{X} H=0$, i.e., $H$ is parallel. Using a frame field $\left(E_{i}\right), 1 \leq i \leq 5$ with $E_{5}$ in $D^{\perp}$ and the rest in $D$, one can write $H=\gamma J E_{5}$ for some constant $\gamma$. Also the equation $h(X, Y)=g(X, Y) H$ gives $h\left(E_{i}, E_{i}\right)=\gamma J E_{5}$, and $h\left(E_{i}, E_{j}\right)=0$ for $i \neq j$. Note that in this case

$$
\begin{aligned}
\left(\bar{\nabla}_{E_{i}}{ }^{h)\left(E_{j}, E_{k}\right)}\right. & =\stackrel{1}{\nabla}_{E_{i}}\left(E_{j}, E_{k}\right)-h\left(\nabla_{E_{i}} E_{j}, E_{k}\right)-h\left(E_{j}, \nabla_{E_{i}} E_{k}\right) \\
& =E_{i} g\left(E_{j}, E_{k}\right) H=0 \text { for all } i, j .
\end{aligned}
$$

where we have used the equation $h(X, Y)=g(X, Y) H$ in the second equality. This means that $M$ has parallel second fundamental form. Then using Theorem 1 we obtain Theorem 2 .

PROOF OF THEOREM 3. Let $\{X, Y, J X, J Y, Z\}$ be an orthonormal frame for $T M$ where $X, Y \in D$ and $Z \in D^{\perp}$. Since $M$ is a hypersurface we know that the above frame diagonalizes $A$.

Therefore one can write

and

$$
h(Z, Z)=\alpha J Z, h(X, X)=\beta J Z, h(J X, J X)=\gamma J Z, h(Y, Y)=\delta J Z, h(J Y, J Y)=\eta J Z
$$

$$
h(Z, X)=h(Z, J X)=h(Z, Y)=h(Z, J Y)=h(X, J X)=h(X, Y)=h(X, J Y)=h(Y, J Y)=0
$$

where $\alpha, \beta, \gamma, \delta, \eta$ are smooth functions on $M$. Then using Guass equation (1.3) we get

$$
S(Z, Z)=R(X, Z, Z, X)+R(J X, Z, Z, J X)+R(Y, Z, Z, Y)+R(J Y, Z, Z, J X)=4 c+\alpha(\beta+\gamma+\delta+\eta)
$$

Similarly

$$
\begin{aligned}
& S(X, X)=4 c+\beta(\alpha+\gamma+\delta+\eta) \\
& S(J X, J X)=4 c+\gamma(\alpha+\beta+\delta+\eta) \\
& S(Y, Y)=4 c+\delta(\alpha+\beta+\gamma+\eta) \\
& S(J Y, J Y)=4 c+\eta(\alpha+\beta+\delta+\eta)
\end{aligned}
$$

Since $M$ is Einstein we have i.e.,

$$
S(Z, Z)=S(X, X)=S(J X, J X)=S(Y, Y)=S(J Y, J Y)=\text { constant }
$$

$$
\alpha(\beta+\gamma+\delta+\eta)=\beta(\alpha+\gamma+\delta+\eta)=\gamma(\alpha+\beta+\delta+\eta)=\delta(\alpha+\beta+\gamma+\eta)=\eta(\alpha+\beta+\gamma+\delta)=\text { const } .
$$

(iii) 
We shall show that $\alpha, \beta, \gamma, \delta$ and $\eta$ are constants. From the above equations we have:

$$
\begin{array}{ll}
\alpha(\gamma+\delta+\eta)=\beta(\gamma+\delta+\eta), & \beta(\alpha+\delta+\eta)=\gamma(\alpha+\delta+\eta) \\
\gamma(\alpha+\beta+\eta)=\delta(\alpha+\beta+\eta), & \delta(\alpha+\beta+\gamma)=\eta(\alpha+\beta+\gamma)
\end{array}
$$

We seek all solutions for this system. One obvious solution is $\alpha=\beta=\gamma=\delta=\eta=$ const. The other possible solutions are the following cases:

(a) $\gamma+\delta+\eta=\alpha+\delta+\eta=\alpha+\beta+\eta=\alpha+\beta+\gamma=0$

In this case we have $\alpha=\gamma=\eta=$ const. and $\delta=\beta$ considering (i) and (iv) with $\delta=\beta$ we get $\delta=\beta=\alpha=$ const. or $\delta=\beta=-2 \alpha=$ const. So for this case $\alpha, \beta, \gamma, \delta, \eta$ are constants.

(b) $\alpha=\beta, \alpha+\delta+\eta=\alpha+\beta+\eta=\alpha+\beta+\gamma=0$, i.e., $\alpha=\beta=\delta$ and $\eta=\gamma$. Using (ii) and (v) with $\alpha \doteq \beta=\delta$ we get $\eta=\gamma=\alpha=$ const. or $\eta=\gamma=-2 \alpha=$ const., i.e., $\alpha, \beta, \gamma, \delta, \eta$ are constants.

(c) $\alpha=\beta=\gamma, \alpha+\beta+\eta=\alpha+\beta+\gamma=0$, i.e., $\alpha=\beta=\gamma=\eta$. Using (i) and (iv) with this last equation we get $\alpha=\beta=\gamma=\eta=\delta=$ const. (Note that in case $\alpha=\beta=\gamma=\delta=0$, then $M$ is totally geodesic and hence $\delta=0$ ).

(d) $\alpha=\beta=\gamma=\delta, \alpha+\beta+\gamma=0$, i.e., $\alpha=\beta=\gamma=\delta=0$. Following the note in (c) we have $\eta=0$.

Therefore in all cases $\alpha=\beta=\gamma=\delta=\eta=$ const. We conclude that $H=\alpha J Z$ where $\alpha$ is constant and thus $\nabla_{V} \frac{1}{V} H=0$ for any $V \in \mathscr{S}(M)$, i.e., $M$ is an extrinsic sphere.

\section{REFERENCES}

1. BEJANCU, A., CR-submanifolds of a Kaehlerian manifold I, Proc. Amer. Math. Soc. 69 (1978), 135-142.

2. DILLEN, F.; VERSTRADEN, L. \& VRANCKEN, L., On almost complex surfaces of the nearly Kaehler 6-sphere II, Kodai Math. J. 10 (1978), 261-271.

3. EJIRI, N., Totally real submanifolds in 6-sphere, Proc. Amer. Math. Soc. 83 (1981), 759-763.

4. FUKAMI, T. \& ISHIHARA, S., Almost Hermitian structure on $S^{\mathbf{6}}$, Tohoku Math. J. 7 (1955), 151-156.

5. GRAY, A., Almost complex submanifolds of six sphere, Proc. Amer. Math. Soc. 20 (1969), 277-279.

6. SEKIGAWA, K., Some CR-submanifolds in a 6-dimensional sphere, Tensor N.S. 41 (1984), 13-19. 


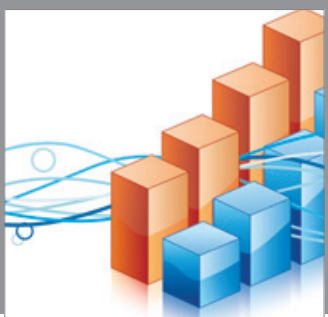

Advances in

Operations Research

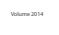

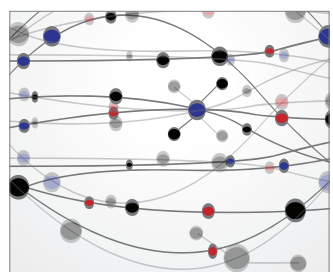

\section{The Scientific} World Journal
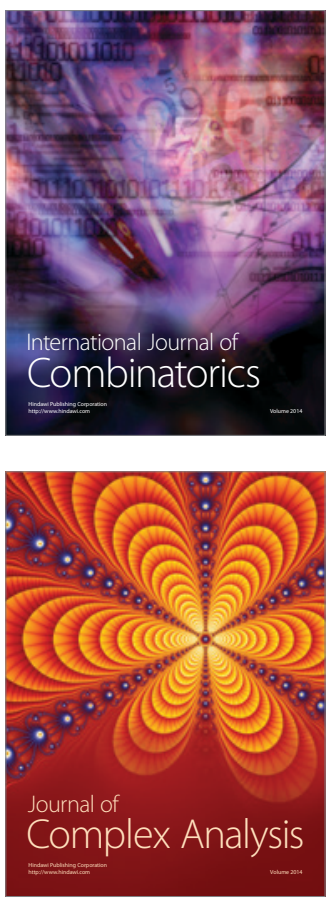

International Journal of

Mathematics and

Mathematical

Sciences
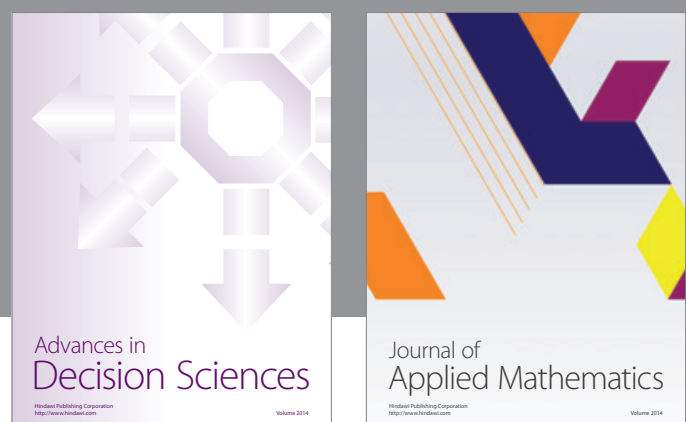

Journal of

Applied Mathematics
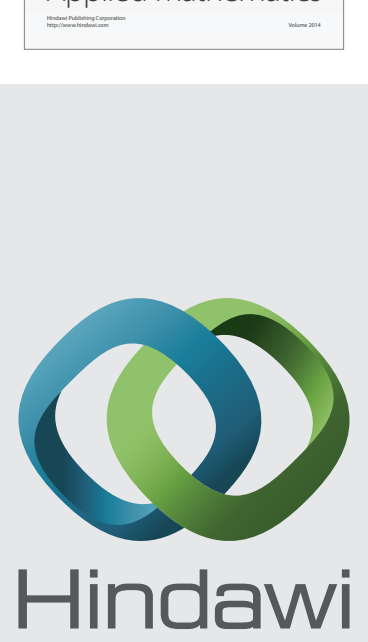

Submit your manuscripts at http://www.hindawi.com
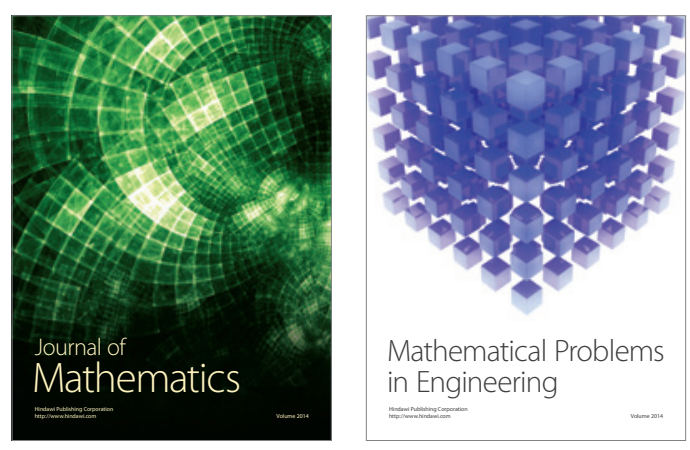

Mathematical Problems in Engineering
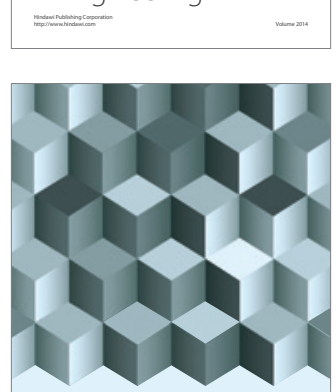

Journal of

Function Spaces
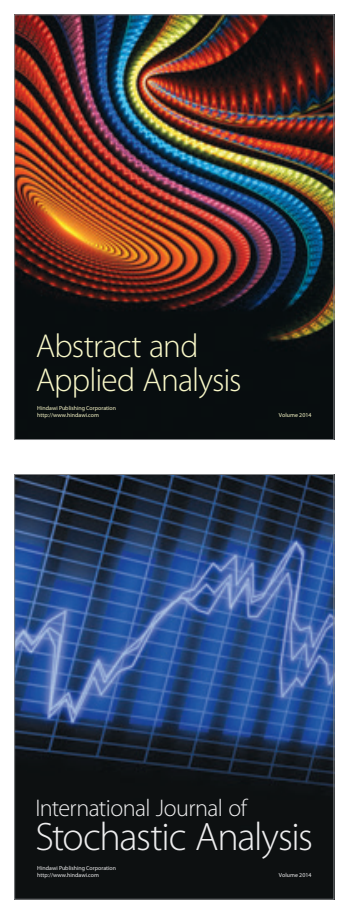

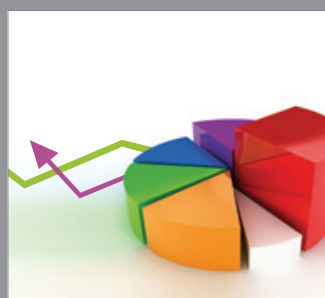

ournal of

Probability and Statistics

Promensencen
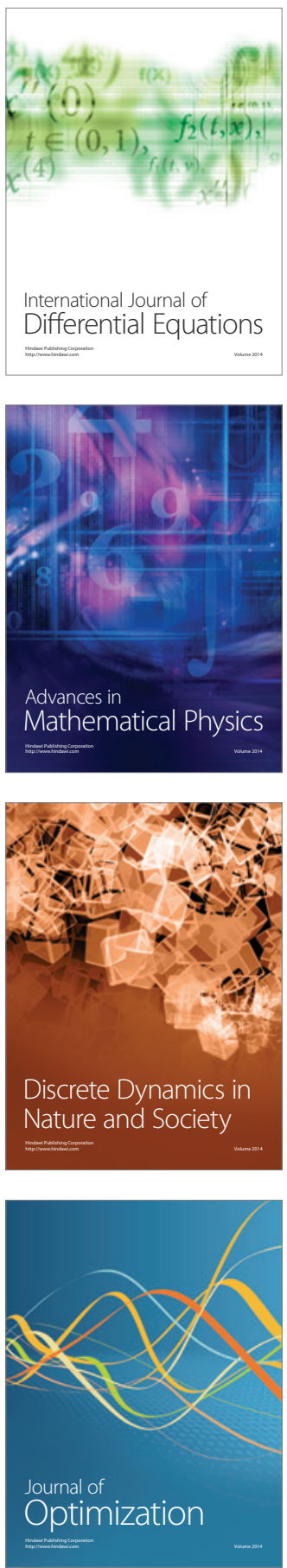